\title{
Türkiye'de Buxus sempervirens L. (Buxaceae) ve Cupressus macrocarpa Hartw. (Cupressaceae) üzerinde yeni kayıt zararlı bir akar; Eurytetranychus buxi (Garman)(Acari: Tetranychidae)*
}

\author{
A first report of Boxwood Spider Mite, Eurytetranychus buxi (Garman)(Acari: \\ Tetranychidae) on Buxus sempervirens L. (Boxwood Tree) and Cupressus macrocarpa \\ Hartw (Macrocarpa Goldcrest) for Turkey
}

\section{Sultan ÇOBANOĞLU ${ }^{1 * *}$}

\author{
Tuğba ERDOĞAN ${ }^{1}$
}

\section{Ayşe YEŞiLAYER ${ }^{2}$}

\section{Summary}

The boxwood spider mite Eurytetranychus buxi (Garman) (Acari: Tetranychidae) was collected on boxwood bushes, Buxus sempervirens L. (Buxaceae) in Ankara and on cypress Cupressus macrocarpa Hartw. (Cupressaceae) in Istanbul. This species is the first report for mite fauna of Turkey. The data on distribution, damage, biology and control of this mite is presented.

Key words: Boxwood spider mite, Eurytetranychus buxi, Tetranychidae, Cupressaceae, Turkey.

\section{Özet}

Ankara'dan şimşir Buxus sempervirens L. (Buxaceae) ve İstanbul'dan Limon servi (Cupressus macrocarpa Hartw. (Cupressaceae) üzerinden toplanan şimşir akarı Eurytetranychus buxi (Garman) (Acari: Tetranychidae), Türkiye akar faunası için ilk kayıt olarak belirlenmiştir. Akarın dünyadaki yayılış alanları, biyolojisi, zarar şekli ve mücadelesi ile ilgili bilgiler sunulmuştur.

Anahtar sözcükler: Eurytetranychus buxi, Şimşir akarı, Cupressaceae, Tetranychidae, Türkiye.

\footnotetext{
* Bu çalışma "European Union Foundation FP7 IRSES Grant No: 269133" projesinin bir parçasıdır.

${ }^{1}$ Ankara Üniversitesi Ziraat Fakültesi Bitki Koruma Bölümü, 06110-Ankara

${ }^{2}$ Gazi Osmanpaşa Üniversitesi Ziraat Fakültesi Bitki Koruma Bölümü,Tokat

** Sorumlu yazar (Corresponding, author) e-mail: Scobanoglu@ankara.edu.tr

Alınış (Received): 19.08.2014 Kabul ediliş (Accepted): 03.11.2014
} 
Türkiye'de Buxus sempervirens L. (Buxaceae) ve Cupressus macrocarpa Hartw. (Cupressaceae) üzerinde yeni kayıt zararlı bir akar; Eurytetranychus buxi (Garman)(Acari: Tetranychidae)

\section{Giriş}

Eurytetranychus Oudemans günümüze kadar 17 türü belirlenmiş olan Tetranychinae alt familyasında yer alan küçük bir cinsdir (Migeon \& Dorkeld, 2006-2013). Eurytetranychus cinsi içinde beş tür Avrupa ve yakın çevresindeki ülkelerde saptanmıştır.

Eurytetranychus admes (Pritchard \& Baker, 1955), Fransa ve Macaristan (Migeon, 2003; Migeon \& Dorkeld, 2006-2013); Eurytetranychus buxi (Garman), Azerbaycan, Belçika; Ermenistan, Fransa, Gürcistan, Hollanda, İran, İrlanda, İtalya; İngiltere, İspanya, Macaristan, Polonya, Portekiz ve Ukrayna (Khalil-Manesh, 1973; Jeppson et al., 1975; Carmona \& Dias, 1980; Mitrofanov et al., 1987; Bernini et al., 1995; Dobosz et al., 1995; Navajas et al., 1996; Bolland et al., 1998; Bolland, 2001; Witters et al., 2003; Migeon \& Dorkeld, 2006-2013); Eurytetranychus furcisetus Wainstein, Macaristan (Bozai, 1974); Kazakistan ve Kırgızistan (Mitrofanov et al., 1987); Eurytetranychus piceus (Löyttyniemi), Finlandiya (Loyttyniemi, 1973); Eurytetranychus ulmi Wang, Çin ve İtalya (Bernini et al., 1995; Migeon \& Dorkeld, 2006-2013)' da tespit edilmiştir.

Eurytetranychus buxi (Garman) (Acari: Tetranychidae) şimşir, Buxus sempervirens L. (Buxaceae), bitkisinin yaprak ve sürgün gibi tüm yeşil aksamı ile beslenebilmektedir. Şimşir yapraklarının alt yüzeyinde ana damara yakın kısmında koloni oluşturmaktadırlar. Eurytetranychus türlerinin çoğu konukçusuna özelleşmiştir (Jeppson et al., 1975).

Eurytetranychus cinsine bağlı türler genel olarak Cupressaceae türlerinde Calocedrus decurrens Pritchard \& Baker, Cupressocyparis leylandii (Jacks. \& Dallim) Juniperus sp., J. communis L., J. sabina L., J. virginiana L. (Cupressaceae), Picea abies (L.) ve P. glauca (Moench) (Pinaceae) gibi ibreli ağaçlara özelleşmiştir (Migeon, 2003). Bununla birlikte E. buxi'nin genel olarak park ve yeşil alanlarda bulunan şimşir bitkilerinde zararlı olduğu bilinmektedir (Jeppson et al., 1975; Raupp et al., 1992; Jagdale et al., 2002).

Türkiye'de Eurytetranychus cinsi ve Eurytetranychus buxi türünün tespit edildiğine dair bir kayıt bulunmamaktadır. Ankara'dan alınan şimşir yapraklarında tespit edilirken dünyada bilinen özel konukçusu dışında İstanbul ilinde "limon servi" Cupressus macrocarpa Hartw. (Cupressaceae) üzerinde yetiştirilen alanlarda saptanan şimşir akarı E. buxi, Türkiye faunası için ilk kayıt olarak verilmektedir.

\section{Materyal ve Yöntem}

Eurytetranychus buxi kolonileri ile yoğun bulaşık Ankara (Ankara Üniversitesi Ziraat Fakültesi Bahçesi) şimşir bitkilerinden Buxus sempervirens L. (Buxaceae) ve İstanbul' dan, "limon servi" C. macrocarpa Hartw. (Cupressaceae) sürgünleri kesilerek, örnekler alınmış ve laboratuvara getirilmiştir (Şekil 1).

Yaprak ve sürgün örnekleri, Nisan-Mayıs aylarında her bir örnekleme alanına haftada bir gidilerek ağaç ve çalıların çeşitli yön ve seviyelerinden toplanmıştır. Bitki örnekleri Berlese hunisinde ekstrakte edilmiş ve akarlar stereomikroskop altında incelenerek \% 70'lik etil alkole toplanmıştır. Örnekler laktofenol ile temizlendikten sonra "Hoyer" ortamında daimi preparatları yapılmıştır (Düzgüneş, 1980). Preparatlar temizlenmesi için $40-50^{\circ} \mathrm{C}$ de 15 gün boyunca etüvde bekletilmiştir.

Örneklerin tanım ve teşhisi Pritchard \& Baker (1955), Tuttle \& Baker (1968), Jeppson et al. (1975) ve Meyer (1987)' den yararlanılarak yapılmıştır.

Zararlı türün tüm biyolojik evrelerinin morfolojileri ve zarar şekli ile ilgili gözlemler ve laboratuvar koşullarında stereomikroskop altında yapılmış orijinal çalışma sonuçlarını yansıtmaktadır. Tüm ölçümler $(\mu \mathrm{m})$ olarak değerlendirilmiştir. Minimum ve maksimum değerler parantez içinde ortalama değerleri takip edecek şekilde verilmiştir. Ölçüm ve fotoğraflarda "Leica SMzZ 2000 Image system" kullanılmıştır. Şimşir 
örnekleri, Ankara (S. Çobanoğlu ve T. Erdoğan), Cupressus macrocarpa örnekleri İstanbul (A. Yeşilayer)' dan toplanmıştır. Tüm örnekler Ankara Üniversitesi Ziraat Fakültesi Bitki Koruma Bölümü akar kolleksiyonlarında muhafaza edilmektedir (S. Çobanoğlu).

\section{Araştırma Sonuçları ve Tartışma}

Tetranychidae-Tetranychinae-Eurytetranychini

Eurytetranychus Oudemans

Bu cins bireylerinde empodium çok küçük ve tırnak şeklindedir. I. bacak tarsusunda "dublex setalar" bulunmaz veya çok sıkı ilişkili olmayıp gevşek yapılıdır. Dişide, tibia III ve IV' de duygu kılları bulunmaz. Para anallar iki çifttir.

Erkekte, "aedeagus" dorsale doğru kıvrılmıştır (Jeppson et al., 1975).

Tip tür: Tetranychus latus Oudemans=Neotetranychus buxi Garman

\section{Eurytetranychus buxi (Garman), Şimşir Akarı}

Sinonimleri: Pritchard \& Baker (1955) ve Migeon \& Dorkeld (2006-2013)'e göre

Eurytetranychus buxi (Garman 1935 ) Ries (1935): 55.

Neotetranychus buxi Garman Ries 1935: 57.

Tetranychus latus Berlese, 1889: 7.

Simplinychus buxi (McGregor 1950),

Eurytetranychus latus Ewing 1932: 14.

Eurytetranychus latus Oudemans 1931: 224.

Tip Konukçu: Buxus sempervirens L. (Buxaceae).

Tip lokalite: Amerika Birleşik Devletleri.

İncelenen materyal: Ankara-Merkez, 26 우, 1 L, 09.V.2014; 18 우, 1 đิ, 20.V.2014; 20 o우, 27.V.2014; 15 웅, 29.V.2014, Buxus sempervirens (Buxaceae); İstanbul-Bayrampaşa, 2 우우, 23.X.2007, Cupressus macrocarpa (Cupressaceae).

Yumurta: Yumurtaları yuvarlak, hassas görünümlü, mat sarı renkli, üzerinde ince çizgiler halinde yivler bulunur (Şekil 1). Yumurtaların limon sarısı renginde ve üzerinde belirgin desenlenmeler bulunduğu belirtilmektedir (Jeppson et al.,1975).

Larva: Koyu yeşil kahverengimsidir. Yumurtalar 6-10 gün içinde açılarak yeşil renkli larvalar ortaya çıkmaktadır. Nimf dönemleri koyu yeşil, yeşilimsi, kahverengi renklidir (Jeppson et al.,1975).

Dişi $(n=10)$ : Kahverengimsi kırmızı renklidir. Dişi idiosoma uzunluğu: ortalama 400 (191-529) $\mu m$, genişliği ise ortalama 294 (143-402) $\mu$ m' dur. Erginler ovalimsi geniş yumuşak vücutludur, hemen hemen yuvarlak ve yassıdırlar (Şekil 1). 


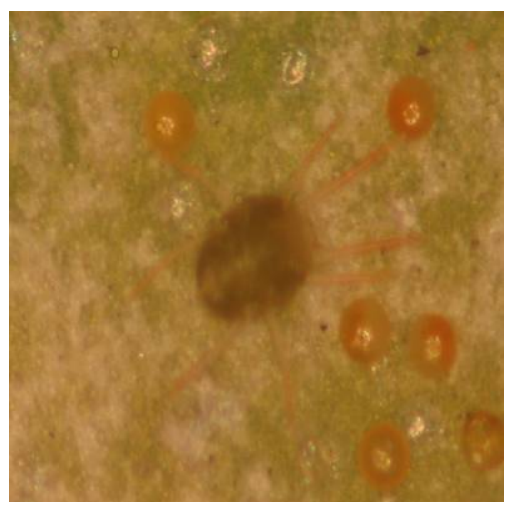

Şekil 1. Eurytetranychus buxi (Garman, 1935), yumurta ve dişilerinin görünümü (Özgün).

Dorsal vücut kılları nispeten uzun olup bir sonraki kıl kökü ile olan mesafe uzunluğu kadardır. Vücut yüzeyindeki kıllar çok küçük tüberkıllar üzerine yerleşmiş fırça gibi hafif tüylü (Lanceolate) görünümdedir (Şekil 2 a, b).

Dorsal vücut kılları; Propodosomal P1 19,13; P2 27,87; P3 17,59; c1 12,86; c2 26,48; d1 16,48; d2 28,18; e1 26,16; e2 30,95; f1 31,28; f2 33,43 um. uzunluktadır (Şekil 2 b).

Gnathosama: (n=10): Uzunluğu ortalama 86 (82-114) $\mu$ m'dir. Güçlü stylet bulunur ve mandibular plate üzerine yerleşmiştir. Mandibular plate geniş ve yuvarlak görünümlüdür (Şekil 2 a). Palpus tarsusunda güçlü bir tırnak bulunur ve sensillum silindirik, uzun görünümlüdür. Genişliğinin uzunluğuna oranı: 8,85 / 2,49=3,55 (n=6) (Şekil 2 e). Peritrem küçük bir şişkinlikle sonlanır.

Bacaklar, ince orta uzunlukta ve vücuttan daha açık renktedir. Bacaklar sarımtırak renklerde görülürler. Empodium çok küçük ve tırnak şeklindedir. Empodium tennent kılların yarısı boyundadır. I. bacak tarsusunda dublex setalar çok sıkı ilişkili olmayıp bir çifttir. Birbirlerinden mesafeli yerleşmişlerdir (Şekil 2c).

Bacak boyları; I. bacak 180 (172-185); II. bacak 128 (112-139); III. bacak 135 (132-138); IV. bacak 123 (103-148) $\mu \mathrm{m}$. ' dir.

Tarsus III ve IV'de duygu kıllarının bulunmadığı belirtilmektedir (Pritchard \& Baker, 1955).

E. buxi, empodiumunda küçük tırnak bulunur. Her iki cinsiyette de bir "dublex setae" bulunur ve bazen "dublex" kıllar serbest gevşek yapılı görünümlüdürler (Jeppson et al., 1975).

Dişide iki çift anal setae bulunur (Şekil 2d).

Eurytetranychus neobuxi Meyer'in dorsal kıllarının uzun ve tüylü yapıda olduğunu ve palpus tarsusunda yer alan sensillum'un uzun görünümlü olduğunu ifade edilmektedir (Meyer, 1987).

Erkek ( $n=1)$ : Vücut yapısı ve kılların yerleşimi dişiye benzerdir. Erkeğin idiosoma uzunluğu: 346 $\mu \mathrm{m}$, genişliği ise $268 \mu \mathrm{m}$ ' dir. Palpusta bulunan sensillum (Spinneret) uzunluğunun genişliğine oranı 6,48 / $1,78=3,64$ 'dür. Adeagus dorsale yönelik, karakteristik ve ana gövdeye yaklaşık dik açı yapacak şekilde görülür (Şekil 3a). Duplex seta bulunmaz (Şekil 3b). 

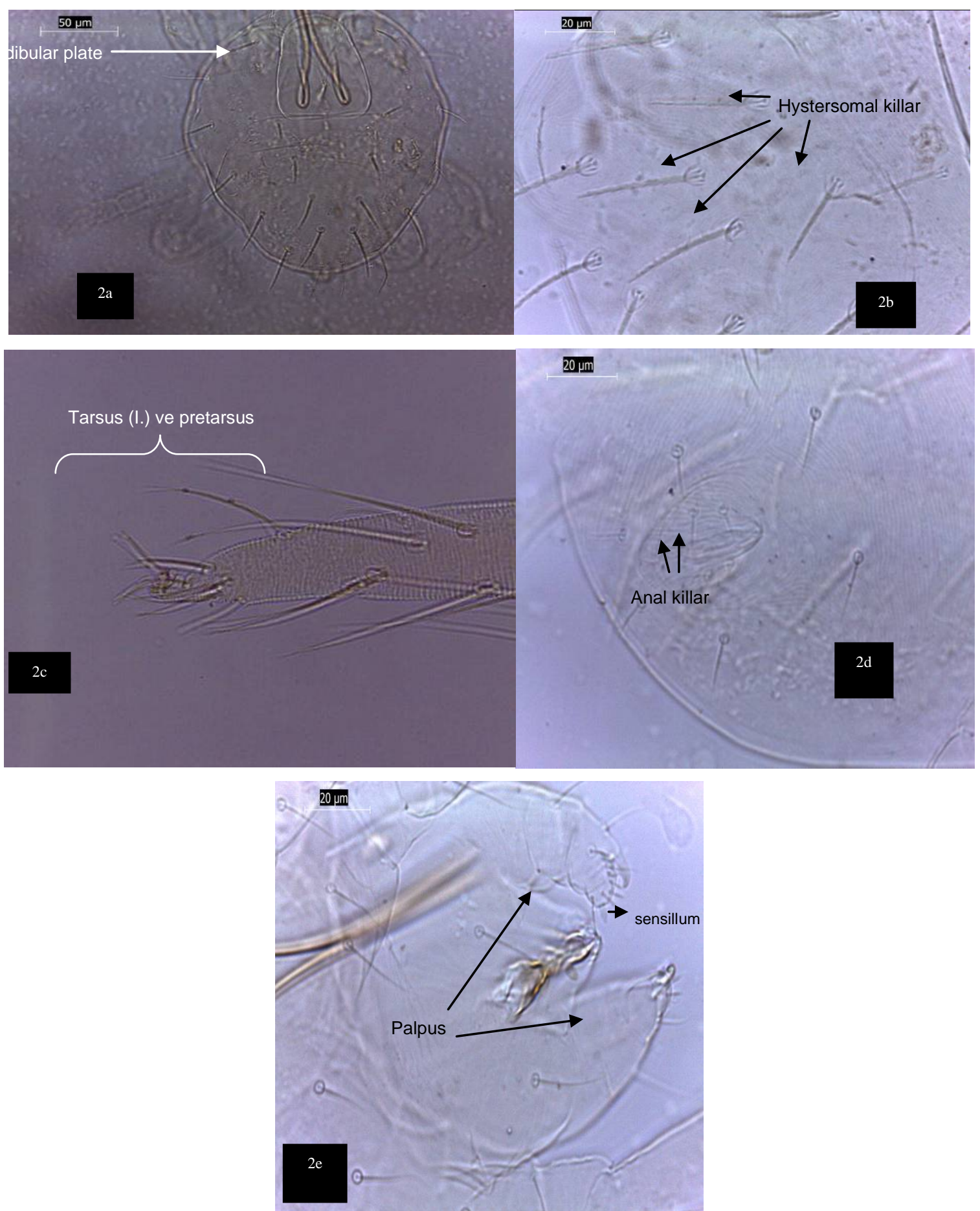

Şekil 2. Eurytetranychus buxi (Garman, 1935) Dişi; a) Mandibular plate ve idiosoma; b) Dorsal kıllar; c)Tarsus (I.) ve pretarsus; d) Anal plate; e) Palpus ve sensillum. (Özgün) . 
Türkiye'de Buxus sempervirens L. (Buxaceae) ve Cupressus macrocarpa Hartw. (Cupressaceae) üzerinde yeni kayıt zararlı bir akar; Eurytetranychus buxi (Garman)(Acari: Tetranychidae)

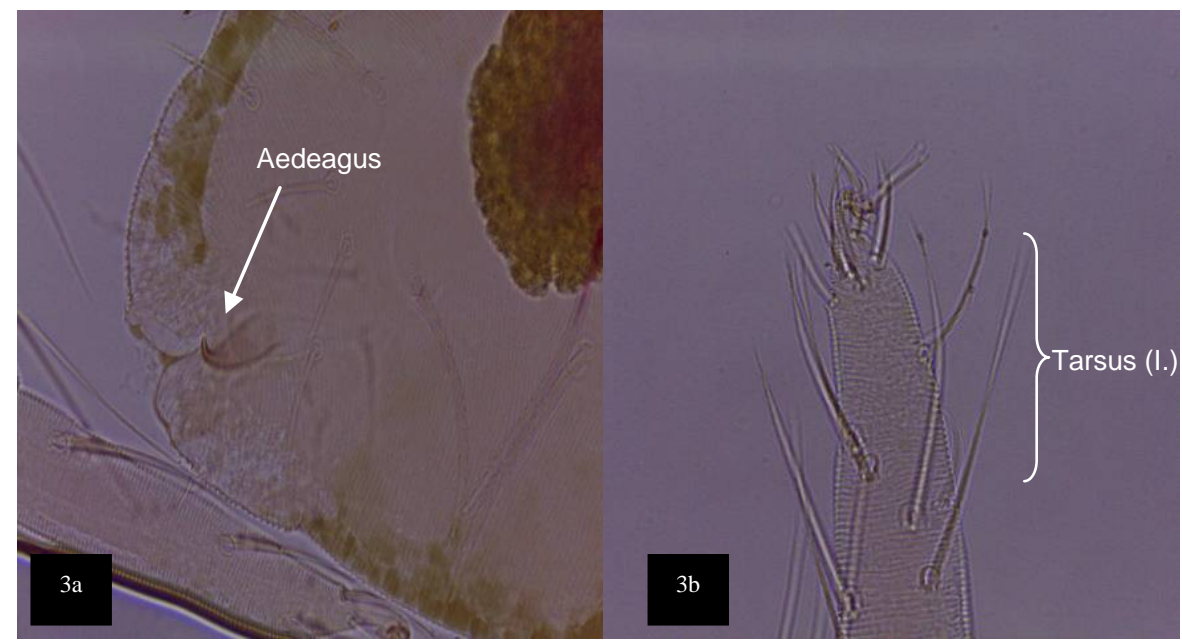

Şekil 3. Eurytetranychus buxi (Garman, 1935) Erkek a) Aedeagus; b) Tarsus (I.) (Özgün) .

E. neobuxi, erkeğinde palpus sensillum'unun uzunluğu genişliğinin üç katı ve aedeagus yapısının yaklaşık doksan derece açıyla dorsale yönelik yapıda olduğu belirtilmektedir (Meyer, 1987). Belirtilen özellikler Türkiye'den saptanan türe çok benzemekte ve onun özelliklerini teyit eder niteliktedir.

Dünyada konukçuları: Buxaceae, Buxus microphylla (Pritchard \& Baker, 1955); Buxus sempervirens (Pritchard \& Baker, 1955; Navajas et al., 1996; Witters et al., 2003); Buxus sp. (Pritchard \& Baker, 1955) üzerinde saptanmıştır.

Zararı: Eurytetranychus buxi şimşir bitkilerinin yaprak ve sürgün gibi tüm yeşil aksamı ile beslenebilmektedir. Şimşir yapraklarının alt yüzeyinde ana damar boyunca koloni oluşturmaktadırlar. Tüm hareketli dönemleri yaprak ve genç sürgünlerde öz suyu emerek zararlı olurlar. Zarar sonucu sarı, beyazımsı noktalar halinde lekeler meydana gelir, sürgün gelişimi durur, yoğunluk artıkça yapraklar önce sararır daha sonra gümüşi renk alarak sonunda kahverengiye döner ve dökülür. Zarar öncelikle yaşı sürgün ve yaşlı yapraklarda yoğun olarak görülür. Bu tür diğer tetranychidler gibi yoğun ağ oluşturmaz. Şimşirlerde akar popülasyonları yoğun olduğunda çok zayıf ağ ördükleri gözlenmiştir. Yoğunluk arttığında yaprakların üst yüzünde de beslenebilmektedir (Şekil 1, 4, 5).

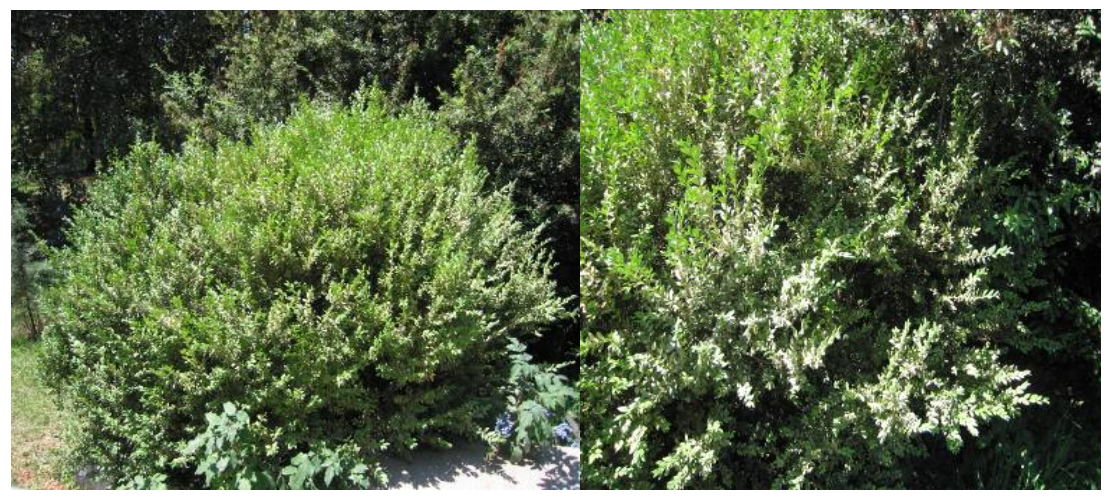

Şekil 4. Eurytetranychus buxi (Garman, 1935). Şimşirde oluşturduğu renk açılmaları (Özgün). 


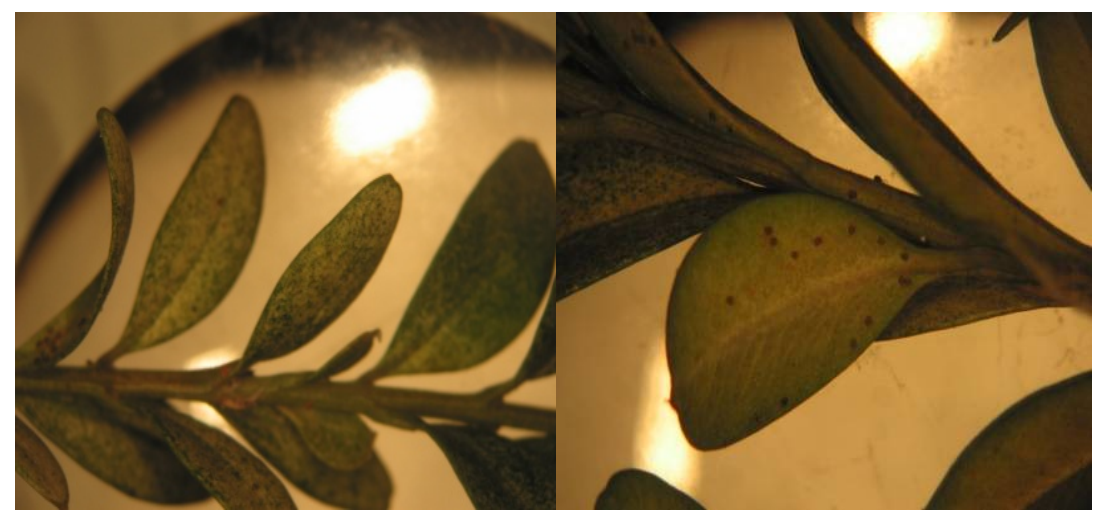

Şekil 5. Eurytetranychus buxi (Garman, 1935). Şimşir yapraklarında oluşturduğu zarar şekli ve koloniler (Özgün).

Eurytetranychus buxi, şimşir bitkilerini tercih etmekte ve yaprakların üzerinde sarı lekeler oluşturmaktadır. Popülasyonun yoğun olduğu durumlarda yapraklar tamamen bronz renk almakta ve dökülmektedir. Bitki genel olarak zayıf görünüme neden olmaktadır (Jeppson et al., 1975).

\section{Dünyadaki Dağılımı:}

Nearctic: Amerika Birleşik Devletleri (Pritchard \& Baker, 1955; Migeon \& Dorkeld, 2006-2013).

Palearctic: Azerbaycan, Ermenistan, Gürcistan, Ukrayna (Mitrofanov et al., 1987); Belçika (Bolland et al., 1998; Witters et al., 2003); Büyük Britanya (Jeppson et al., 1975); Fransa (Navajas et al., 1996); İran (Khalil-Manesh, 1973); Hollanda, İrlanda, İtalya, Macaristan, Portekiz, İspanya, Polonya (Migeon \& Dorkeld, 2006-2013).

Tartışma: Eurytetranychus buxi kışı yumurta olarak geçirir. Yılda ortalama 8 döl meydana getirdiği ve yüksek sıcaklık ve düşük nemin bu akarın gelişimini teşvik ettiği belirtilmektedir (Jeppson et al., 1975). Zararı daha çok ilkbahar aylarında görülmektedir. Mevsim ilerledikçe zarar oranı düşmektedir. Kışlayan yumurtalar Eylül ve Ekim aylarında bırakılmakta, Nisan ortalarında yumurta açılımıyla birlikte üç çift bacaklı larva meydana gelmektedir. Sakin dönem olarak adlandırılan dinlenme evresinin arkasından iki nimf devresi ve ergin görülmektedir. Dişi olgunlaşır olgunlaşmaz yumurtlama başlar ve dişi başına 25-30 yumurta bırakılır. Toplam gelişme süresi 18-21 gün arasında değişmektedir (Jeppson et al., 1975). E. neobuxi türünün farklı popülasyonları arasında önemli ölçüde varyasyon olduğu ve aynı popülasyon bireyleri arasında dahi farklılıklar gözlendiği kaydedilmektedir (Meyer, 1987). Bu nedenle farklı popülasyonlarda tür düzeyinde yapılacak çalışmalarda yanılgıya düşülebileceği dikkate alınmalıdır.

Doğal düşmanları arasında; E. buxi, örneklerinin toplandığı bahçelerde yoğun olarak Phytoseiidae türleri saptandığı belirtilmektedir. Diğer doğal düşmanları ise Stethorus punctum (LeConte) (Coleoptera: Coccinellidae), Chrysoperla carnea (Stephens) (Neuroptera: Chrysopidae; Coniopterygidae), Neuropter türleri (Neuroptera) ve ceccidomyid larvaları (Diptera: Ceccidomyiidae) olarak belirtilmiştir (Huffaker \& Messenger, 1976; Helle \& Sabelis, 1985, Dicke et al., 1999, Roda et al., 2000; Rott \& Ponsonby, 2000; Reddy, 2001, Roy et al., 2002, Roy et al., 2003). Ülkemiz akar faunası için ilk kayıt niteliğinde olan bu türün biyolojisi, doğal düşmanları ve ülkemizdeki dağılımı konusunda ayrıntılı çalışmaların yapıımasının yararlı olacağı kanısındayız.

\section{Teşekkür}

Eurytetranychus buxi (Garman) teşhisinin doğrulanmasında yardımcı olan Prof. Dr. Eddie Ueckermann (ARC-Plant Protection Research Institute, Queenswood, Pretoria, South Africa)'a teşekkür ederiz. Bu çalışma, "European Union Foundation FP7 IRSES Grant No.: 269133 project" kapsamında desteklenmiştir. 
Türkiye'de Buxus sempervirens L. (Buxaceae) ve Cupressus macrocarpa Hartw. (Cupressaceae) üzerinde yeni kayıt zararlı bir akar; Eurytetranychus buxi (Garman)(Acari: Tetranychidae)

\section{Yararlanılan Kaynaklar}

Bernini, F., M. Castagnoli \& R. Nannelli, 1995. Arachnida Acari. Minelli, A., Ruffo, S. and La Posta, S., Checklist delle species della fauna italiana, Commission of the European Communities, 24: 24-66.

Bolland H. R., J. Gutierrez \& C. H. W. Flechtmann, 1998. World Catalogue of the Spider Mite Family (Acari: Tetranychidae). Brill Academic Publishers, Leiden (NL). Bull. Agr. Res. Sta. Rehovoth, 21: 1- 63.

Bolland, H. R., 2001. Mites (Acari: Tetranychidae and Phytoseiidae) from the Tatra mountains in Slovakia, with special remarks on Tetranycopsis hystriciformis Reck. International Journal of Acarology, 27: $225-227$.

Bozai, J., 1974. Contributions to the knowledge of the Tetranchoid fauna of Hungary (Acari) Folia Entomologica Hungarica, 27: 5-7.

Carmona, M. M. \& J. C. S. Dias, 1980. O complexo Acarina nas culturas portuguesas. I. Congresso Português Fitiatria e Fitofarmacologia, Lisboa, Portugal, 2: 97-115.

Dicke M., R. Gols, D. Ledeking \& M. A. Posthumus, 1999. Jasmonic acid and herbivory differentially induce carnivore-attracting plant volatiles in lima bean plants. Journal of Chemical Ecology, 25: 1907.

Dobosz, R., A. Skorupska \& C. Blaszak, 1995. The appearance of spider mites (Tetranychidae) in parks of Poznan. Boczek, J. and Ignatowicz, S., Materialy z Sympozjum na temat: "Osiagniecia Akarologii w Polsce, 39-42 pp.", Siedlce, 26-27 wresnia 1995, Komitet Ochrony Roslin Polska Akademia Nauk, 285 pp.

Düzgüneş, Z., 1980. Küçük Arthropodların Toplanması Saklanması Mikroskobik ve Preparatlarının Hazırlanması. T. C. Gıda Tarım ve Hayvancılık Bakanlığı Zirai Mücadele ve Zirai Karantina Müdürlüğü Yayınları, Ankara, 77s.

Helle, W. \& M. W. Sabelis, 1985. Spider Mites: Their Biology, Natural Enemies and Control. Vol. 1B. Elsevier Scientific Publishers, New York. 449 pp.

Huffaker, C. B. \& P. S. Messenger, 1976. Theory and Practice of Biological Control. Academic Press. London. 788 pp.

Jagdale, G. B., N. Somasekhar, P. S. Grewal \& M. G. Klein, 2002. Suppression of plant - parasitic nematodes by application of live and dead infective juveniles of an entomopathogenic nematode, Steinernema carpocapsae, on boxwood (Buxus spp.). Biological Control, 24: 42-49.

Jeppson, L.R., H. H. Keifer \& E. W. Baker, 1975. Mites Injurious To Economic plants. University of California Press, Berkeley, xxiv+614pp.

Khalil-Manesh, B., 1973. Phytophagous mite fauna of Iran. Entomologie et Phytopathologie Appliquées, 30-38.

Loyttyniemi, K., 1973. Eurytetranychus piceus sp. n. (Acarina, Tetranychidae) with notes on its life history. Annales Entomologici Fennici, 39: 119-123.

Meyer, M. K. P. 1987. African Tetranychidae (Acari: Prostigmata). Entomology Mem. Department of Agriculture Techechnical Service, Republic of South Africa, 69, 175 pp.

Migeon, A., 2003. Eurytetranychus admes: un nouvel acarien tétranyque sur les cyprès en France. Phytoma-La Défense des Végétaux, 561: 30-31.

Migeon, A. \& D. Dorkeld, 2006-2013. Spider Mites Web: a comprehensive database for the Tetranychidae. http://www.montpellier.inra.fr/CBGP/spmweb (05.11. 2014)

Mitrofanov, V.I., Z. I. Strunkova \& I. Z. Livshits, 1987. Keys to the Tetranychid Mites (Tetranychidae, Bryobiidae) Fauna of the USSR and Adjacent Countries. SSR, I.o.Z.a.P.E.N.P.T. Dushanbe, Donish: 224 pp.

Navajas, M., J. Gutierrez, J. Lagnel \& P. Boursot, 1996. Mitochondrial cytochrome oxidase I in tetranychid mites: a comparison between molecular phylogeny and changes of morphological and life history traits. Bulletin of Entomological Research, 86: 407-417.

Oudemans, A.C., 1931. Acarologische aanteekeningen CVII. Ent. Ber., Amst. 8(178): 221-236.

Pritchard, A. E. \& E. W. Baker, 1955. A Revision of the Spider Mite Family Tetranychidae. Memoirs Series, San Francisco, Pacific Coast Entomological Society, 2, 472 pp.

Raupp, M. J., C. S. Koehler \& J. A. Davidson, 1992. Advances in implementing integrated pest management for woody landscape plants. Annual Review of Entomology 37: 561-85. 
Reddy, G. V. P., 2001. Comparative effectiveness of an integrated pest management system and other control tactics for managing the spider mite Tetranychus ledeni (Acari: Tetranychidae) on eggplant. Experimental and Applied Acarology 25: 985-92.

Ries, D.T.,1935. A new mite (Neotetranychus buxi n. sp. Garman) on boxwood. Journal of Economic Entomology, 28: 55-62.

Roda, A., J. Nyrop, M. Dicke \& G. English-Loeb, 2000. Trichomes and spider mite webbing protect predatory mite eggs from intraguild predation. Oecologia, 125: 428-35.

Rott, A. S. \& D. J. Ponsonby, 2000. The effects of temperature, relative humidity and host plant on the behaviour of Stethorus punctillum Weise (Coleoptera: Coccinellidae) as a predator of the twospotted spider mite, Tetranychus urticae Koch (Acari: Tetranychidae): Biocontrol, 45: 155-164.

Roy, M., J. Brodeur \& C. Cloutier, 2002. Relationship between temperature and developmental rate of Stethorus punctillum (Coleoptera: Coccinellidae) and its prey Tetranychus mcdanieli (Acari: Tetranychidae). Environmental Entomology, 31: 177-87.

Roy, M., J. Brodeur \& C. Cloutier, 2003. Effect of temperature on intrinsic rates of natural increase ( $\mathrm{rm}$ ) of a occinellid and its spider mite prey. Biocontrol, 48: 57-72.

Tuttle D. M. \& E. W. Baker, 1968. Spider Mites of Southwestern United States and a Revision of the Family Tetranychidae. TheUniversity of Arizona Press, Tuscon-Arizona, U.S.A., 143 pp.

Witters, J., H. Casteels \& G. D. Bondt, 2003. Diagnostic acarological research at the Department of Crop Protection in 2002-2003. Parasitica, 59: 107-111. 